\title{
Wave-Driven Mass Loss: A mechanism for late-stage stellar eruptions
}

\author{
Josh Shiode and Eliot Quataert \\ UC Berkeley, Department of Astronomy, \\ 601 Campbell Hall \#3411, UC Berkeley, \\ Berkeley, CA, USA 94720-3411
}

\begin{abstract}
During the late stages of stellar evolution in massive stars (carbon fusion and later), the fusion and neutrino luminosities in the core of the star exceed the Eddington luminosity. This can drive vigorous convective motions which in turn excite a super-Eddington flux in internal gravity waves. We show that an interesting fraction of the energy in excited gravity waves can, in some cases, convert into sound waves as the gravity waves propagate (tunnel) towards the stellar surface. The subsequent dissipation of the sound waves can unbind up to several $M_{\odot}$ of the stellar envelope. This wave-driven mass loss can explain the existence of extremely large stellar mass loss rates just prior to core-collapse, which are inferred via circumstellar interaction in some core-collapse supernovae (e.g., SNe 2006gy and PTF 09uj).
\end{abstract}

Keywords. stars: mass loss, winds, outflows; supernovae: general

\section{Introduction}

The interaction between an outgoing supernova shock and $\sim 1-10 \mathrm{M}_{\odot}$ of material ejected in the $\sim$ year prior to core-collapse, and thus ending up $\sim 100$ AU from the progenitor, can explain the most optically luminous supernovae (SNe) yet detected (e.g., Smith \& McCray 2007), including, e.g., SN 2006gy (Smith et al. 2007) and perhaps the emerging class of hydrogen-poor superluminous SNe (SLSNe) discussed by both Quimby and Chornock in these proceedings. However, the giant eruptive mass-loss events preceding these SNe lack a plausible mechanism.

During this last $\sim$ year of evolution, the fusion and neutrino luminosities in the core of a massive star significantly exceed the Eddington luminosity (see, e.g. Woosley et al. 2002, for a review). We propose that in some SLSNe progenitors, gravity waves, excited by strong convection in the burning core, can tunnel out to the stellar envelope, and deposit enough energy to drive the inferred prodigious pre-supernova mass-loss (Quataert \& Shiode 2012).

\section{Convection and Wave Excitation During Late Stages of Massive Star Evolution}

Convection excites a gravity-wave (g-mode) luminosity given by $\mathrm{L}_{\text {wave }} \sim \mathcal{M}_{\text {conv }} \mathrm{L}_{\text {conv }}$, where $\mathcal{M}_{\text {conv }}$ is the convective Mach number $\left(v_{\text {conv }} / c_{\text {sound }}\right)$ and $L_{\text {conv }}$ is the convective luminosity (Goldreich \& Kumar 1990). This energy is predominantly in g-modes with spherical harmonic degree, $\ell$, and frequency, $\omega$, determined by the convective mixing length and timescales, so that the spherical degree $\ell \sim r / H$ and frequency $\omega \sim v_{\text {conv }} / H$, where $r$ is the local radius and $H$ the local pressure scale height (Kumar et al. 1999). During core $\mathrm{O} / \mathrm{Ne}$ burning, $\sim 1$ yr before core-collapse, for a typical massive star, waves will be excited in a buoyantly-stable layer overlying the convective core, with $\mathrm{L}_{\text {wave }} \sim$ $0.01 \mathrm{~L}_{\text {conv }} \sim 10^{-3} \mathrm{~L}_{\text {nuc }} \gg \mathrm{L}_{\text {Eddington }}$. 


\section{Tunneling vs. Local Damping}

Convectively excited gravity-waves can propagate as acoustic waves (p-modes) in the stellar envelope if they can tunnel through the evanescent region that separates the gand p-mode propagation cavities. The width of this region and effective strength of the potential barrier are defined by the $\ell$ and $\omega$ of the mode. Waves can tunnel if they do not reach amplitudes comparable to their wavelength in the cavity where they are excited (i.e., break) and their time to damp by neutrino radiation is longer than the time to tunnel. These conditions are given by (respectively):

$$
\begin{aligned}
k_{r} \xi_{r} & \sim \Lambda^{3 / 2}\left(\frac{N}{\omega}\right)^{3 / 2}\left(\frac{\mathrm{F}_{\text {wave }}}{\rho r^{3} \omega^{3}}\right)^{1 / 2} \lesssim 1, \\
t_{\nu} & \sim \frac{t_{\text {nuc }}}{100}>t_{\text {tunnel }} \sim \frac{r_{\text {in }}}{v_{g}}\left(\frac{r_{\text {out }}}{r_{\text {in }}}\right)^{2 \Lambda},
\end{aligned}
$$

where $\mathrm{F}_{\text {wave }}$ is the wave energy flux, $\Lambda^{2}=\ell(\ell+1), t_{\text {nuc }}$ the nuclear burning timescale ( $\sim$ year $), \rho$ the density, $r$ the radius, $k_{r}$ the radial wavenumber, $\xi_{r}$ the wave's radial amplitude, $N$ the Brunt-Väisälä frequency, $v_{g}$ the g-mode group velocity, and $r_{i n}$ and $r_{\text {out }}$ the inner and outer radii of the tunneling region. Waves that tunnel will deposit their energy in the envelope when the radiative diffusion time across a wavelength is comparable to the group travel time over a scale height, which can be written as

$$
L_{\mathrm{rad}} \gtrsim L_{\mathrm{damp}} \equiv \frac{4 \pi r^{2} \rho c_{\mathrm{sound}}^{3}}{\left(k_{r} H\right)^{2}},
$$

where the symbols are as given above.

\section{Conclusions}

We find that high frequency modes, $\omega \gtrsim 3 v_{\text {conv }} / H$, of low spherical degree, $\ell \lesssim$ few, are most likely to tunnel out to the stellar envelope. Modes of lower frequency likely do not satisfy condition 3.1, and are thus more likely to induce mixing locally above the convective core. If most of the super-Eddington flux in gravity waves meets both criteria 3.1 and 3.2, they will tunnel out to the stellar envelope and deposit their energy at a radius given by 3.3 , where it is capable of unbinding up to several solar masses of material during the year prior to core-collapse. If instead, the excitation is preferentially towards modes of lower frequency, these waves will likely break locally and induce mixing above the convective core (see simulations by Meakin \& Arnett 2006, 2011 for possible examples of this). An outstanding question is understanding what stellar parameters (mass, rotation, metallicity, age) are the most susceptible to wave-driven mass loss or mixing. This depends on both the precise internal structure of massive stars and the power-spectrum of internal gravity waves excited by stellar convection.

\section{References}

Arnett, W. D. \& Meakin, C. 2011, ApJ, 733, 78

Goldreich, P. \& Kumar, P. 1990, ApJ, 363, 694

Kumar, P., Talon, S., \& Zahn, J.-P. 1999, ApJ, 520, 859

Meakin, C. A. \& Arnett, D. 2006, ApJL, 637, L53

Quataert, E. \& Shiode, J. 2012, MNRAS, in press

Smith, N. \& McCray, R. 2007, ApJL, 671, L17

Smith, N., et al. 2007, ApJ, 666, 1116

Woosley, S. E., Heger, A., \& Weaver, T. A. 2002, Reviews of Modern Physics, 74, 1015 